\title{
Separability And Aggregation of Equivalence Relations *
}

\author{
Dinko Dimitrov $^{\dagger}$, Thierry Marchant ${ }^{\ddagger}$, and Debasis Mishra ${ }^{\S}$
}

October 18, 2010

\begin{abstract}
We provide axiomatic characterizations of two natural families of rules for aggregating equivalence relations: the family of join aggregators and the family of meet aggregators. The central conditions in these characterizations are two separability axioms. Disjunctive separability, neutrality, and unanimity characterize the family of join aggregators. On the other hand, conjunctive separability and unanimity characterize the family of meet aggregators. We show another characterization of the family of meet aggregators using conjunctive separability and two Pareto axioms, Pareto ${ }^{+}$and Pareto $^{-}$. If we drop Pareto ${ }^{-}$, then conjunctive separability and Pareto ${ }^{+}$characterize $^{-}$ the family of meet aggregators along with a trivial aggregator.
\end{abstract}

KEYWORDS: aggregation; equivalence relations; separability; unanimity; Pareto axiom

JEL Classification: C0; D0

${ }^{*}$ Authors are listed alphabetically. The authors are thankful to an anonymous referee and Bernard Monjardet for detailed comments on the paper. Special thanks also go to Alan Miller and Arunava Sen for their comments.

${ }^{\dagger}$ Saarland University, Email: dinko.dimitrov@mx.uni-saarland.de

${ }^{\ddagger}$ Ghent University, Email: thierry.marchant@ugent. be

${ }^{\S}$ Corresponding author, Indian Statistical Institute, Email: dmishra@isid.ac.in 


\section{INTRODUCTION}

The theory of aggregating individual preferences into a social preference relation was initiated by the seminal work of Arrow (1951) ${ }^{1}$. Arrow's impossibility theorem inspired many scholars to apply his approach to other environments as well. For instance, in many situations a planner is often required to classify a finite set of candidates into "equivalence classes", where two candidates belong to the same class if and only if they are assumed to be equivalent. Such a classification of candidates into disjoint equivalence classes is called an equivalence relation (partition), and it is often based on the various attributes the candidates may have. The objective is to then aggregate these equivalence relations of different attributes and form a holistic equivalence relation - a problem studied in detail in various papers such as (Mirkin, 1975), (Wilson, 1978), (Fishburn and Rubinstein, 1986), (Leclerc, 1984), (Rubinstein and Fishburn, 1986), (Barthélemy et al., 1986), (Neumann and Norton Jr., 1986), and (Barthélemy, 1988). The critical difference of this problem from the Arrovian framework of individual preference aggregation is that an equivalence relation does not rank the equivalence classes it contains.

We give an example to illustrate the applicability of this model.

- Partitioning Households of a City. A city planner wants to form an aggregate equivalence relation of the households of a city. It first collects data from households about their employment, religion, family etc. Here, the candidates are households and the attributes are employment, religion, family. The collected data enables the city planner to form an equivalence relation of the households for every attribute. He now needs to generate an aggregate equivalence relation from these equivalence relations. Such an aggregate equivalence relation may help form development policies, and improve targeting of such policies.

It is natural that out of the entire set of possible attributes, the planner only considers a subset of the attributes relevant for constructing the aggregate partition of candidates. The planner may then think of two natural, but somewhat dual, ways to aggregate the equivalence relations in each attribute into one equivalence relation. It can consider two candidates to be equivalent if and only if they are equivalent in each of the relevant attribute. On the other hand, it can take the other extreme view by considering an aggregator which somehow respects the partitioning under every relevant attribute. In this case, it may consider two candidates to be equivalent if they are equivalent under any of the relevant attributes. Other applications of this type of aggregation includes a school wanting to classify its set of students based on various attributes to better target its activities, a group of experimental researchers trying to classify a data-set (population in a village, living beings in a forest etc.) based on various attributes to design its experiment, a political party classifying various constituencies on various attributes to better plan its campaign etc.

\footnotetext{
${ }^{1}$ Some recent pedagogic proofs of Arrow's impossibility theorem appear in Perote-Pena and Piggins (2002) and Geanakoplos (2005).
} 
In this paper we focus on such aggregation of equivalence relations, consider these two natural families of aggregation rules, and axiomatically characterize them. To start with, let us consider an example with three candidates say $a, b$, and $c$, and two attributes, say 1 and 2. Objects $a$ and $b$ are equivalent but candidate $c$ is different in terms of attribute 1 . Objects $a$ and $c$ are equivalent but $b$ is different in terms of attribute 2. We will now describe the two families of aggregators using this example. For both families, we first identify a non-empty subset of attributes, called the decisive attributes. For each possible choice of decisive attributes, we then define two aggregators. Thus, each family of such aggregators has $2^{n}-1$ members, where $n$ is the number of attributes.

The first aggregator puts two candidates in the same equivalence class if and only if each decisive attribute puts them in the same equivalence class. Thus, it reflects the consensus view of the decisive attributes and is a member of the family of meet aggregators. In the example above, if we take both the attributes as decisive, then the equivalence relation given by this meet aggregator puts each candidate in a separate equivalence class.

Each aggregator in the second family of rules we discuss has an entirely different approach in aggregation. It takes the union (or join) of the equivalence classes of every decisive attribute. In some sense, it tries to "satisfy" each decisive attribute, and belongs to the family of join aggregators. In the example above, if we take both attributes as decisive, the join aggregator puts $a$ and $b$ together due to attribute 1 . Also, it puts $a$ and $c$ together due to attribute 2 , and hence, all the three candidates form an equivalence class.

\subsection{Our Contribution}

We provide characterizations for the family of meet aggregators and for the family of join aggregators. The central theme of our characterizations is separability. Separability can be thought as the counterpart of additivity and consistency axioms in this model. Additivity and consistency has been recognized as a central axiom in cooperative game theory, and used in various models (for example, Shapley (1953) uses additivity and Hart and Mas-Colell (1989) use consistency to axiomatize the Shapley value). We motivate our versions of separability axioms in Section 3.1.

We use two (dual) versions of separability axioms. Conjunctive separability requires that if we combine two profiles of equivalence relations using the meet operation, then the aggre-

gator must output the same equivalence relation on this combined data as the equivalence relation obtained by taking the meet of the equivalence relations produced by the aggregator for each profile of equivalence relations. Analogously, disjunctive separability requires that if the combination of equivalence relations according to both approaches is done using the join operation, then it must produce the same equivalence relation. These two axioms reflect two consistent ways of decentralizing the process of aggregation.

Besides these two separability axioms, we use some standard axioms from the aggregation theory literature. We start with three different Pareto-type axioms. The first one, Pareto ${ }^{+}$, 
says that if two candidates are in the same equivalence class according to every attribute, then the aggregator must put them in the same equivalence class. Analogously, we define the Pareto $^{-}$axiom, which requires that if two candidates are in different equivalence classes according to every attribute, then the aggregator must put them in different equivalence classes. Pareto ${ }^{+}$is satisfied by both types of aggregators, while Pareto ${ }^{-}$is satisfied by the meet aggregators but not by the join aggregators. Finally, we consider also a third Paretotype axiom, unanimity, which is satisfied by the aggregators in both families. Unanimity requires that if the equivalence relation according to every attribute is the same, the aggregator must output this very equivalence relation. The combination of Pareto $^{+}$and Pareto $^{-}$ implies unanimity.

The next standard axiom we use is neutrality. It requires that if we construct a new profile of equivalence relations from a given profile of equivalence relations by permuting the set of candidates, then the outcome of the aggregator at the new profile must be the same permutation applied to the outcome of the old profile. Hence, the names of the candidates should not matter to the aggregator.

Finally, we use an axiom called non-triviality. An aggregator is called trivial if it outputs the equivalence relation where all the candidates are put in the same equivalence class for every profile of equivalence relations. An aggregator satisfies non-triviality if it is not a trivial aggregator.

We show that an aggregator satisfies unanimity, neutrality, and disjunctive separability if and only if it is one of the join aggregators. We obtain an almost dual characterization of the family of meet aggregators. An aggregator satisfies unanimity and conjunctive separability if and only if it is one of the meet aggregators. Neutrality is implied by conjunctive separability and unanimity (or Pareto ${ }^{+}$). We show that one can replace unanimity by Pareto $^{+}$and Pareto $^{-}$in the characterization of the meet aggregators.

Pareto $^{-}$is not satisfied by the join aggregators when $m \geq 3$, where $m$ is the number of candidates. A natural task is then to explore the possibility of dropping the Pareto ${ }^{-}$axiom in the characterization of the family of meet aggregators. We show that we do not get a significantly larger set of aggregators if we drop this axiom. In particular, an aggregator satisfies Pareto ${ }^{+}$and conjunctive separability if and only if it is one of the meet aggregators or the trivial aggregator. Hence, using non-triviality in place of Pareto $^{-}$along with Pareto $^{+}$and conjunctive separability characterizes the family of meet aggregators. No such characterization is possible for the family of join aggregators.

Using our characterizations, we can conclude that the families of join and meet aggregators essentially differ by two different notions of separability. These families contain (almost) dual aggregators and our characterizations reflect this duality. 


\subsection{Relation to Prior Work}

The literature on aggregating equivalence relations started with the works of Mirkin (1975), Leclerc (1984), Barthélemy et al. (1986), Fishburn and Rubinstein (1986), and Neumann and Norton Jr. (1986). In their work, Rubinstein and Fishburn (1986) discuss a general model of preference aggregation which covers the aggregation of equivalence relations, while Fishburn and Rubinstein (1986) consider explicitly the family of meet aggregators. To our knowledge, there is no work which discusses the family of join aggregators.

Fishburn and Rubinstein (1986) use Pareto ${ }^{+}$, Pareto ${ }^{-}$, and binary independence to characterize the family of meet aggregators. Binary independence is an axiom in the spirit of Arrow's independence of irrelevant alternatives. It requires that the aggregated equivalence relation between any two candidates must depend only on the equivalence relations between these two candidates in every attribute. Our characterization of the family of meet aggregators replaces binary independence by conjunctive separability for $m \geq 3$. Is binary independence weaker than conjunctive separability? We give examples to illustrate that neither of them imply the other. However, we show that conjunctive separability and binary independence are equivalent axioms (for $m \geq 3$ ) in the presence of Pareto ${ }^{+}$.

Our results for the family of meet aggregators are slightly tighter than the results in Fishburn and Rubinstein (1986). First, the binary independence axiom used in Fishburn and Rubinstein (1986) has no bite when $m=2$. This is not the case for conjunctive separability, and as a result, we get a characterization which works for all $m$. Second, when $m \geq 3$, we show that Pareto ${ }^{-}$in the characterization of Fishburn and Rubinstein (1986) can be weakened to non-triviality.

Independent of our work, Chambers and Miller (2010) derive a characterization of the meet aggregators using conjunctive separability, upper unanimity, and non-imposition. This is similar to our Theorem 4 - while upper unanimity is implied by Pareto ${ }^{+}$, non-impositiion implies non-triviality.

Another related strand of the aggregation literature considers environments in which every individual has a view about how a society he is a member of should be partitioned into classes (see for example, Houy (2007); Dimitrov and Puppe (2010)). A group identity function assigns then to each profile of views a societal decomposition into classes. Hence, this aggregation problem is formally equivalent to the aggregation of equivalence relations and it extends environments in which the number of social groups is assumed to be fixed and their names matter (see for example, Çengelci and Sanver (2010); Dimitrov et al. (2007); Houy (2007); Kasher and Rubinstein (1997); Miller (2008); Samet and Schmeidler (2003), among others). It is worth noting that the specific features of the group identification problem allows one to introduce liberalism-type axioms which have no meaning in the more general framework we consider in the present paper; for instance, Houy (2007) uses such kind of axioms, along with binary independence, to characterize the grand meet aggregator.

The study of the impact of appropriately defined meet and join separability axioms on 
group identification rules was undertaken by Miller (2008) for a specific context, where the number of social groups is fixed. This author then shows that the two requirements basically define a class of one-vote rules, in which one opinion determines whether an individual is considered to be a member of a group.

Another type of aggregation problems in which individuals submit a menu of options are considered by Ahn and Chambers (2008). These authors formulate a disjoint additivity axiom which can be seen as a weaker version of disjunctive separability for the corresponding context, and show that disjoint additivity, anonymity, unanimity, and a monotonicity axiom characterize the grand join aggregator in their model. In the characterizations we provide in this paper, the two separability ideas are formulated as to fit into the more general setting of aggregating equivalence relations and to clearly stress the duality between the aggregators in the considered families.

The rest of the paper is organized as follows. Section 2 discusses the model, the notations, and the general framework, while Section 3 introduces the axioms we use in the paper. We give our characterization of the family of join aggregators in Section 4 and present different characterizations of the family of meet aggregators in Section 5. Section 6 is devoted then to the duality in the characterizations of the two families. We conclude in Section 7 with discussions on the effect of (a) adding anonymity to our characterizations and (b) imposing both forms of separability on an aggregator.

\section{FRAMEWORK}

Let $M=\{a, b, c, \ldots\}$ be a finite set of $m \geq 2$ candidates and $N=\{1,2, \ldots, n\}$ be a finite set of $n \geq 2$ attributes. An equivalence relation on $M$ is a reflexive, symmetric, and transitive binary relation. Formally, $\sim$ is an equivalence relation on $M$ if for all $a, b \in M$, we have

- (Reflexivity) $a \sim a$,

- (Symmetry) $a \sim b \Rightarrow b \sim a$,

- (Transitivity) $a \sim b$ and $b \sim c \Rightarrow a \sim c$.

Equivalently, one can think of an equivalence relation to be a partitioning of set of candidates into equivalence classes, where candidates $a$ and $b$ belong to the same equivalence class in equivalence relation $\sim$ if and only if $a \sim b$.

We will denote the equivalence relation of attribute $i \in N$ as $\sim_{i}$. Thus, $\left(\sim_{1}, \ldots, \sim_{n}\right)$ will denote a profile of equivalence relations. Sometimes, we will refer to a profile $\left(\sim_{1}, \ldots, \sim_{n}\right)$ as $\left(\sim_{i}\right)_{N}$.

One can think of an equivalence relation as being an undirected graph which is transitive. Such a graph will have a node for every candidate, i.e., the set of nodes is $M$. There is an edge between the different candidates $a$ and $b$ if and only if $a \sim b$. We denote the (undirected) 
edge between any two different candidates $a$ and $b$ as $(a, b)^{2}$. Hence, there are three types of equivalence classes: a single node which is not part of any edge (equivalence class with a single candidate), a pair of nodes which are joined by an edge but not part of any cycle (equivalence class with two candidates), and a set of nodes forming a cycle (equivalence classes with more than two candidates).

Let $\mathbb{E}$ be the set of all equivalence relations on $M$. An aggregator is a mapping $F$ : $\mathbb{E}^{n} \rightarrow \mathbb{E}$. So, the aggregator outputs a holistic equivalence relation for every profile of equivalence relations. To simplify notations, sometimes we will write $F\left(\sim_{1}, \ldots, \sim_{n}\right)$ as $\sim$ and $F\left(\sim_{1}^{\prime}, \ldots, \sim_{n}^{\prime}\right)$ as $\sim^{\prime}$, etc.

The following specific types of equivalence relations will be useful in what follows. We will say that an equivalence relation $\sim$ is

- empty, if every equivalence class in $\sim$ contains a single candidate (i.e., the graph corresponding to $\sim$ has no edges),

- single-edged, if one equivalence class in $\sim$ contains two candidates, while every other equivalence class in $\sim$ contains a single candidate (i.e., the graph corresponding to $\sim$ has exactly one edge),

- complete, if $\sim$ contains a single equivalence class consisting of all candidates (i.e., the graph corresponding to $\sim$ is complete).

We define two operations on equivalence relations. The meet of two equivalence relations $\sim$ and $\sim^{\prime}$ is denoted as $\sim \wedge \sim^{\prime}$, and is the largest (with respect to inclusion) equivalence relation contained in both $\sim$ and $\sim^{\prime}$. Consequently, any two candidates $a$ and $b$ are equivalent in $\sim \wedge \sim^{\prime}$ if and only if $a \sim b$ and $a \sim^{\prime} b$. The equivalence relation $\sim \wedge \sim^{\prime}$ can also be seen as $\sim \cap \sim^{\prime}$. The family of meet aggregators is defined as follows. For every non-empty $S \subseteq N$

$$
F_{\wedge}^{S}\left(\sim_{1}, \ldots, \sim_{n}\right)=\wedge_{i \in S} \sim_{i} \quad \forall\left(\sim_{1}, \ldots, \sim_{n}\right) \in \mathbb{E}^{n}
$$

We call $F_{\wedge}^{N}$ the grand meet aggregator.

The join of two equivalence relations $\sim$ and $\sim^{\prime}$ is denoted as $\sim \vee \sim^{\prime}$, and is the smallest (with respect to inclusion) equivalence relation containing both $\sim$ and $\sim^{\prime}$. Consequently, any two candidates $a$ and $b$ are equivalent in $\sim \vee \sim^{\prime}$ if and only if $a \sim b$ or $a \sim^{\prime} b$ or there is $c$ such that $\left(a \sim c\right.$ and $\left.c \sim^{\prime} b\right)$ or $\left(b \sim c\right.$ and $\left.c \sim^{\prime} a\right)$. The equivalence relation $\sim \vee \sim^{\prime}$ can also be seen as the transitive closure of $\sim \cup \sim^{\prime}$. The family of join aggregators is defined as follows. For every non-empty $S \subseteq N$,

$$
F_{\vee}^{S}\left(\sim_{1}, \ldots, \sim_{n}\right)=\vee_{i \in S} \sim_{i} \quad \forall\left(\sim_{1}, \ldots, \sim_{n}\right) \in \mathbb{E}^{n}
$$

\footnotetext{
${ }^{2}$ It is appropriate to denote an undirected edge between $a$ and $b$ by $\{a, b\}$. For convenience, we abuse notation here to denote it as $(a, b)$.
} 
We call $F_{\vee}^{N}$ the grand join aggregator.

Note that the binary operations $\vee$ and $\wedge$ are associative. Hence, for any $S \subseteq N$, the operations $\vee_{i \in S}$ and $\wedge_{i \in S}$ are well-defined. Clearly then, based on the choice of $S$, we will have different meet aggregators and different join aggregators.

\section{Axioms}

In this section, we define the axioms we use later. We start with some well-known axioms in the preference aggregation literature.

Axiom 1 Let $\sigma$ be a permutation of $M$ and for any binary relation $\sim$ define $\sim^{\sigma}$ as $a \sim^{\sigma} b$ if and only if $\sigma(a) \sim \sigma(b)$ for all $a, b \in M$. We say an aggregator $F$ satisfies neutrality if and only if $F\left(\left(\sim_{i}^{\sigma}\right)_{N}\right)=F\left(\left(\sim_{i}\right)_{N}\right)^{\sigma}$ for all profiles of equivalence relations $\left(\sim_{1}, \ldots, \sim_{n}\right)$.

Neutrality requires that the aggregator should not distinguish between candidates based on their names. Similarly, if we do not distinguish between attributes based on their names, then we have the following axiom.

Axiom 2 An aggregator $F$ satisfies anonymity if for any permutation $\rho$ of $N$ and any profile of equivalence relations $\left(\sim_{1}, \ldots, \sim_{n}\right)$ we have $F\left(\left(\sim_{i}\right)_{N}\right)=F\left(\left(\sim_{\rho(i)}\right)_{N}\right)$.

The next two axioms are the equivalent of Pareto axiom in the Arrovian framework.

Axiom 3 An aggregator $F$ satisfies Pareto $^{+}$if and only if for all $a, b \in M$ and for all profiles of equivalence relations $\left(\sim_{1}, \ldots, \sim_{n}\right)$ such that $a \sim_{i} b$ for all $i \in N$, we have $a \sim b$, where $\sim \equiv F\left(\sim_{1}, \ldots, \sim_{n}\right)$.

Axiom 4 An aggregator $F$ satisfies Pareto $^{-}$if and only if for all $a, b \in M$ and for all profiles of equivalence relations $\left(\sim_{1}, \ldots, \sim_{n}\right)$ such that $a \nsim_{i} b$ for all $i \in N$, we have $a \nsim b$, where $\sim F\left(\sim_{1}, \ldots, \sim_{n}\right)$.

It is easy to see that Pareto ${ }^{+}$is satisfied by the family of meet and join aggregators. On the other hand, Pareto ${ }^{-}$is satisfied by the family of meet aggregators but not by the family of join aggregators. To see this, consider an example with two attributes 1 and 2 and three candidates $a, b$, and $c$. Consider the following profile of equivalence relations: $a \sim_{1} b$ but $a \nsim_{1} c$ and $b \nsim_{1} c$ and $a \sim_{2} c$ but $a \nsim_{2} b$ and $c \nsim_{2} b$. Note that $b \nsim_{i} c$ for all $i \in\{1,2\}$. Hence, by Pareto ${ }^{-}, b$ and $c$ should belong to different equivalence classes in the aggregated equivalence relation. Now, consider the grand join aggregator on this profile. It will put $a, b$, and $c$ in one equivalence class.

The following axiom is a weakening of the combination of Pareto ${ }^{+}$and Pareto ${ }^{-}$, and is satisfied by the families of join and meet aggregators. 
AxIOM 5 An aggregator $F$ satisfies unanimity if and only if for all profiles of equivalence relations $\left(\sim_{1}, \ldots, \sim_{n}\right)$ such that $\sim_{1}=\ldots=\sim_{n}$, we have $F\left(\sim_{1}, \ldots, \sim_{n}\right)=\sim_{1}$.

Clearly, the combination of Pareto $^{+}$and Pareto ${ }^{-}$imply unanimity.

The following independence axiom was used in Fishburn and Rubinstein (1986).

Axiom 6 An aggregator $F$ satisfies binary independence if and only if for every $a, b \in M$ and for all pairs of profiles $\left(\sim_{i}\right)_{N}$ and $\left(\sim_{i}^{\prime}\right)_{N}$ such that $a \sim_{i} b$ if and only if $a \sim_{i}^{\prime} b$ for all $i \in N$, we have $a \sim b$ if and only $a \sim^{\prime} b$, where $\sim \equiv F\left(\left(\sim_{i}\right)_{N}\right)$ and $\sim^{\prime} \equiv F\left(\left(\sim_{i}^{\prime}\right)_{N}\right)$.

Finally, we say an aggregator $F$ is trivial if for all profiles $\left(\sim_{i}\right)_{N}, F\left(\left(\sim_{i}\right)_{N}\right)$ is the complete equivalence relation.

Axiom 7 An aggregator satisfies non-triviality if it is not a trivial aggregator.

\subsection{Separability Axioms}

Next, we define our two main axioms on separability. Suppose, the planner does not know the equivalence relation for every attribute. Then, he sends an expert to the "world" to collect this information. In our earlier example, the city planner sends an expert to collect data on households. The expert may take time (a finite number of periods) to collect all the information (we assume that the world is consistent, and does not evolve during the time of collecting these information). Suppose the planner insists on getting a profile of equivalence relations every period. But the expert may not have complete information of the actual profile of equivalence relations in a period (of course, at the end of all periods, the expert does get this actual profile of equivalence relations). The planner may instruct the expert to send the incomplete information as an equivalence relation in every period in the following two natural ways.

1. In any period, the expert reports candidate $a$ and $b$ to be equivalent under attribute $i$ if and only if

(a) he observes in this period $a$ and $b$ to be equivalent under attribute $i$ (i.e., they collect complete information about it in this period), or

(b) he observes in this period $a$ and $c$ to be equivalent under attribute $i$ and $b$ and $c$ to be equivalent under attribute $i$ for some $c \notin\{a, b\}$.

In all other cases, he reports $a$ and $b$ to be not equivalent in this period. So, the interpretation of $a$ and $b$ to be not equivalent according to attribute $i$ in a period is twofold: (i) the expert in this period observed that $a$ and $b$ are not equivalent according to attribute $i$, or (ii) enough information was not collected in this period to conclude whether $a$ and $b$ are equivalent under attribute $i$. Using these instruction, the expert generates a profile of equivalence relations every period. 
It is clear that the final profile of equivalence relations is just the join of the profile of equivalence relations in each period. Suppose the planner intends to use an aggregator every period to form a periodic aggregate equivalence relation. Our first separability axiom requires some consistency between the periodic aggregate equivalence relations and the final aggregate equivalence relation. It says that final aggregate equivalence relation is the join of periodic aggregate equivalence relations.

AxIOM 8 An aggregator $F$ satisfies disjunctive separability if and only if for all pairs of profiles $\left(\sim_{i}\right)_{N}$ and $\left(\sim_{i}^{\prime}\right)_{N}$, we have $F\left(\left(\sim_{i} \vee \sim_{i}^{\prime}\right)_{N}\right)=F\left(\left(\sim_{i}\right)_{N}\right) \vee F\left(\left(\sim_{i}^{\prime}\right)_{N}\right)$.

2. There is another way in which the planner may ask the expert to send incomplete equivalence relations every period. It can ask the expert to report $a$ and $b$ are not equivalent under attribute $i$ if and only if the data has been collected till this period to conclude that $a$ and $b$ are not equivalent under $i$. In all other cases, $a$ and $b$ are reported to be equivalent under $i$. Under these instructions, if no data has been collected for $a$ and $b$ under attribute $i$, then they are reported to be equivalent. It is clear that the final profile of equivalence relations is the meet of the periodic profile of equivalence relations. Again, the planner may use an aggregator to come up with a periodic aggregate equivalence relation. Our second separability axiom requires that the final aggregate equivalence relation is the meet of the periodic aggregate equivalence relations.

Axiom 9 An aggregator $F$ satisfies conjunctive separability if and only if for all pairs of profiles $\left(\sim_{i}\right)_{N}$ and $\left(\sim_{i}^{\prime}\right)_{N}$, we have $F\left(\left(\sim_{i} \wedge \sim_{i}^{\prime}\right)_{N}\right)=F\left(\left(\sim_{i}\right)_{N}\right) \wedge F\left(\left(\sim_{i}^{\prime}\right)_{N}\right)$.

Such separability axioms are primarily motivated by decentralized computation. The periodic profile of equivalence relations can be processed by the expert in a given period using the aggregator of the planner, and the planner can then process the periodic aggregate equivalence relations into a single aggregate equivalence relation. The planner is saved the hassle of taking the meet or join of the profiles of equivalence relations over all periods, and then applying the aggregator himself. Instead, he can decentralize the process by aggregating in each period, and then taking the join or meet (depending on the instructions to the expert) of these periodic aggregate equivalence relations.

\section{Join AgGregators}

In this section, we give a characterization of the family of join aggregators. Our characterization uses the neutrality, unanimity, and disjunctive separability axioms. To give an idea why the characterization works, consider an aggregator which satisfies neutrality, unanimity, and disjunctive separability. Now, consider an example with three attributes $(N=\{1,2,3\})$ 
and four candidates $(M=\{a, b, c, d\})$. Call a profile of equivalence relations a single-edged profile if there is exactly one attribute with a single-edged equivalence relation and all other attributes have empty equivalence relations. The first step in the proof is to show that the

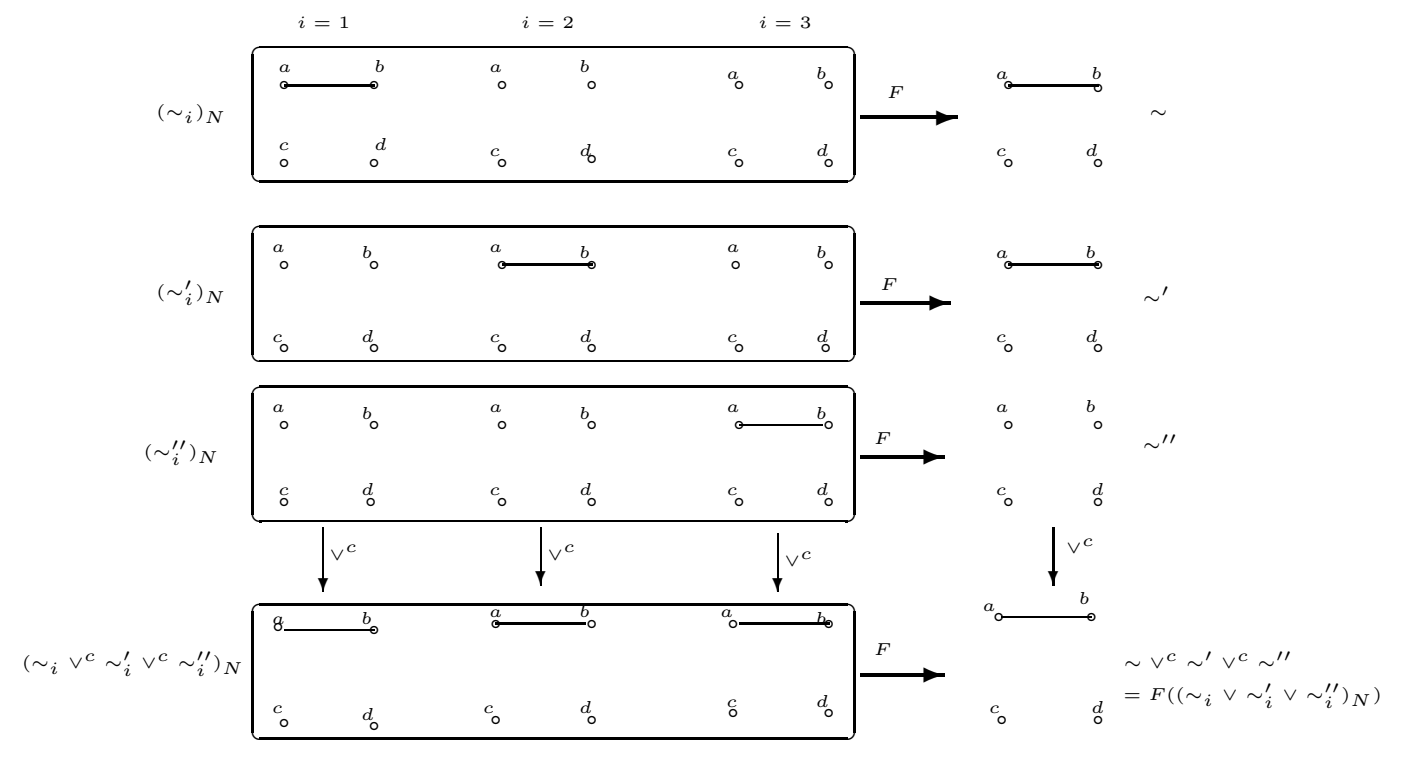

Figure 1: Single-edged profiles

aggregator is a join aggregator for any single-edged profile. To do so, consider the singleedged profiles in Figure 1. Each triplet of relations in a rounded rectangle represents a profile. Each column corresponds to an attribute. The join of these single-edged profiles is a profile (last row), where unanimity axiom can be applied. Hence, the aggregator (applied to the last row) must output the equivalence relation shown in Figure 1. By disjunctive separability, there is some non-empty set of single-edged profiles $\left(\left(\sim_{i}\right)_{N},\left(\sim_{i}^{\prime}\right)_{N}\right.$ or $\left.\left(\sim_{i}^{\prime \prime}\right)_{N}\right)$, such that the corresponding aggregated equivalence relations (resp. $\sim, \sim^{\prime}$ or $\sim^{\prime \prime}$ ) have edge $(a, b)$. Moreover, no other edges must be present in $\sim, \sim^{\prime}$ or $\sim^{\prime \prime}$. Suppose the aggregator outputs a non-empty equivalence relation whenever the single-edged profile has edge $(a, b)$ in attributes 1 or 2 . Denote these attributes as $S^{a b}$. By neutrality, $S^{a b}=S^{c d}=S$ for all $(a, b)$ and $(c, d)$. Thus, the set $S$ collects all decisive attributes and we call it a decisive set. In Figure 1, we have $S=\{1,2\}$ as the decisive set.

The proof is then concluded by observing that any profile of equivalence relations can be decomposed into single-edged profiles whose join is the original profile of equivalence relations. Figure 2 illustrates this using a profile of equivalence relations $\left(\sim_{i}\right)_{N}$ which is the join of 5 (total number of edges in this profile of equivalence relations) single-edged profiles of equivalence relations denoted by $\left(\sim_{i}^{1}\right)_{N},\left(\sim_{i}^{2}\right)_{N},\left(\sim_{i}^{3}\right)_{N},\left(\sim_{i}^{4}\right)_{N}$ and $\left(\sim_{i}^{5}\right)_{N}$. Using the first step of the proof, we know $\sim^{1}, \ldots, \sim^{5}$. The join of these five relations is the cycle with edges $a, b, c$. By disjunctive separability, $F\left(\left(\sim_{i}\right)_{N}\right)=\sim$ must be equal to this cycle, that is, the aggregator must take the join of the equivalence relations in the decisive set.

We now state the theorem and prove it formally. 


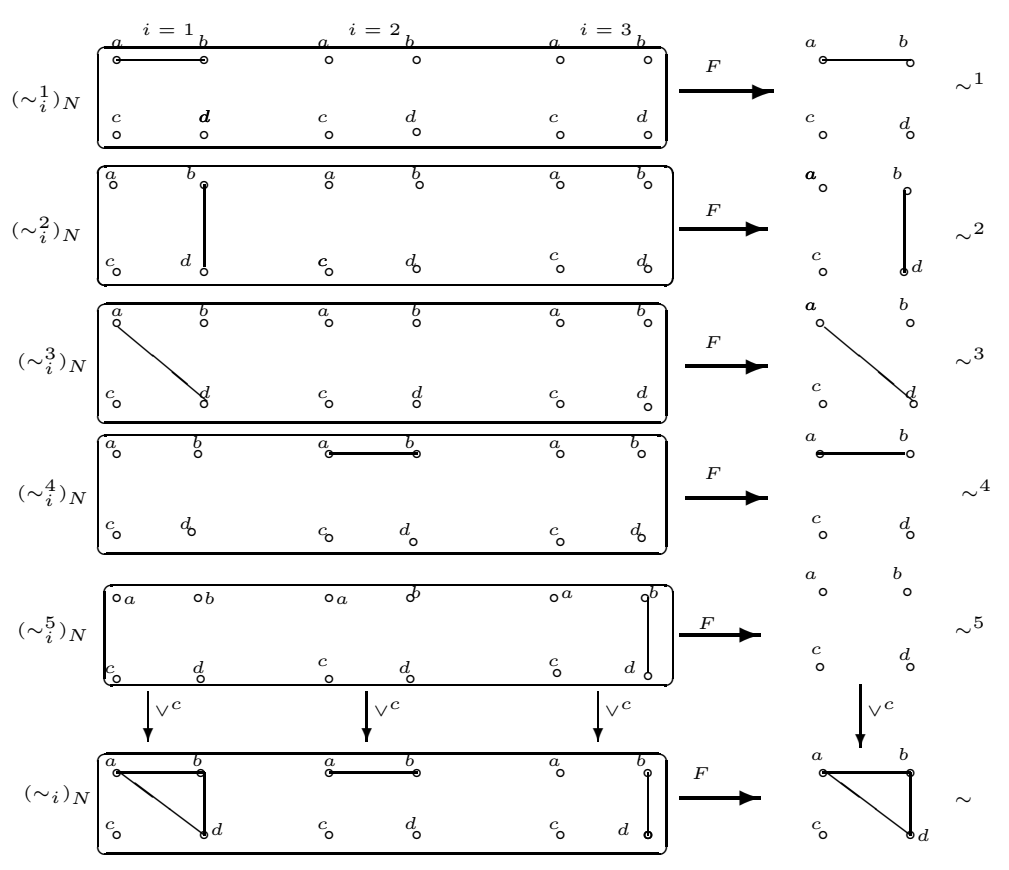

Figure 2: Decomposing a profile into single-edged profiles

THEOREM 1 An aggregator satisfies neutrality, unanimity, and disjunctive separability if and only if it is belongs to the family of join aggregators.

Proof: It is not difficult to see that each join aggregator satisfies neutrality, unanimity, and disjunctive separability. Suppose $F$ is an aggregator which satisfies these three axioms.

Let $\left(\sim_{i}^{k, a b}\right)_{N}$ be a single-edged profile of equivalence relations where edge $(a, b)$ is present in attribute $k$ only, and no other edges are present in any attribute. By construction, the profile $\left(\vee_{k \in N} \sim_{i}^{k, a b}\right)_{N}$ is a profile of equivalence relations as in the statement of the unanimity axiom. Hence, $F\left(\left(\vee_{k \in N} \sim_{i}^{k, a b}\right)_{N}\right)$ is a single-edged equivalence relation with edge $(a, b)$. By disjunctive separability, the edge $(a, b)$ is present in $F\left(\left(\sim_{i}^{k, a b}\right)_{N}\right)$ for at least one $k$, and no other edge is present in $F\left(\left(\sim_{i}^{k, a b}\right)_{N}\right)$. Define the set $S^{a b}$ as follows.

$$
S^{a b}=\left\{k \in N \text { : edge }(a, b) \text { belongs to } F\left(\left(\sim_{i}^{k, a b}\right)_{N}\right)\right\} .
$$

We can also define $S^{c d}$ for all $c, d \in M$ using profiles $\left(\sim_{i}^{k, c d}\right)_{N}$. By neutrality, $S^{a b}=S^{c d}=S$ for all $a, b, c, d \in M$. Call $S$ the set of decisive attributes (the decisive set). Hence, for every single-edged profile, the aggregator is the join over the decisive set.

Now, consider any arbitrary profile of equivalence relations $\left(\sim_{i}\right)_{N}$. We can decompose it into a finite number of single-edged profiles $\left(\sim_{i}^{j}\right)_{N}$, where $j \in\{1, \ldots, l\}$ and $l$ is the number of edges in the profile $\left(\sim_{i}\right)_{N}$. Thus, $\left(\sim_{i}\right)_{N}=\left(\vee_{j=1, \ldots, l} \sim_{i}^{j}\right)_{N}$. By disjunctive separability, $F\left(\left(\sim_{i}\right)_{N}\right)=F\left(\left(\vee_{j=1, \ldots, l} \sim_{i}^{j}\right)_{N}\right)=\vee_{j=1, \ldots, l} F\left(\left(\sim_{i}^{j}\right)_{N}\right)$. Since the aggregator for the single-edged profiles is the join over the corresponding decisive set, it follows that the aggregator over any profile is also the join over that decisive set. The result then follows because the decisive set is non-empty. 
To complete the characterization in Theorem 1, we show that the axioms neutrality, unanimity, and disjunctive separability are independent. The trivial aggregator satisfies neutrality and disjunctive separability, but it fails unanimity. The grand meet aggregator satisfies neutrality and unanimity but fails disjunctive separability.

To show that neutrality is not implied by unanimity and disjunctive separability, we construct the following aggregator.

EXAmple 1 Fix two candidates $a, b \in M$. For a given profile, $\left(\sim_{i}\right)_{N}$, define a binary relation $I$ as follows. We have $a I b$ if and only if $a \sim_{1} b$. For any $(c, d) \neq(a, b)$, we have $c I d$ if and only if $c \sim_{i} d$ for some $i \in N$. Define $F\left(\left(\sim_{i}\right)_{N}\right)$ as the symmetric and transitive closure of $I$.

The aggregator in Example 1 satisfies unanimity and disjunctive separability, but fails neutrality. The fact that it fails neutrality is clear (it distinguishes between pairs $(a, b)$ and other pairs). Also, it is easy to see that it satisfies unanimity. To show that it satisfies disjunctive separability requires some effort. We do this in the Appendix.

Also, note that if $m=2$, then every aggregator satisfies neutrality. So, Example 1 works only when $m \geq 3$.

\section{Meet Aggregators}

In this section, we set out to give a characterization of the family of meet aggregators using conjunctive separability. Our aim is to give a characterization which is analogous to the characterization of the family of join aggregators in Theorem 1. Fishburn and Rubinstein (1986) provided the following characterization.

TheOREM 2 (Fishburn and Rubinstein (1986)) Suppose $m \geq 3$. An aggregator satisfies Pareto ${ }^{+}$, Pareto $^{-}$, and binary independence if and only if it belongs to the family of meet aggregators.

Our first characterization of the meet aggregators replaces binary independence in Theorem 2 by conjunctive separability. Unlike Theorem 2, this characterization works also for $m=2$.

TheOREM 3 An aggregator satisfies Pareto ${ }^{+}$, Pareto ${ }^{-}$, and conjunctive separability if and only if it belongs to the family of meet aggregators.

Proof: It is easy to see that each meet aggregator satisfies Pareto $^{+}$, Pareto $^{-}$, and conjunctive separability. Consider an aggregator $F$ which satisfies these three axioms. We how that it must be a meet aggregator. We do the proof in two cases.

CASE 1: Suppose $m \geq 3$. We use the following lemma for this case. 
LEMMA 1 If an aggregator F satisfies Pareto ${ }^{+}$and conjunctive separability, then it satisfies binary independence.

Proof: Suppose $F$ is an aggregator which satisfies Pareto $^{+}$and conjunctive separabilty. We do the proof in two steps.

Step 1: Fix $a, b \in M$ and consider any profile of equivalence relations $\left(\sim_{i}\right)_{N}$. We then consider the equivalence relation $\left(\sim_{i}^{\prime}\right)_{N}$, which is defined by deleting all edges except the edge $(a, b)$ from $\sim_{i}$ for all $i \in N$ (so for all $i \in N,(a, b)$ is an edge in $\sim_{i}$ if and only if $(a, b$ ) is an edge in $\sim_{i}^{\prime}$, but for all $(c, d) \neq(a, b),(c, d)$ is not an edge in $\left.\sim_{i}^{\prime}\right)$. Now, consider the profile of equivalence relations $\left(\sim_{i}^{\prime \prime}\right)_{N}$, where for all $i \in N, \sim_{i}^{\prime \prime}$ is a single-edged equivalence relation with edge $(a, b)$. Note the following:

- $\sim_{i} \wedge \sim_{i}^{\prime \prime}=\sim_{i}^{\prime}$ for all $i \in N$ and

- by Pareto ${ }^{+}$, the edge $(a, b)$ belongs to $F\left(\left(\sim_{i}^{\prime \prime}\right)_{N}\right)$.

Using conjunctive separability, we can conclude that the edge $(a, b)$ belongs to $F\left(\left(\sim_{i}\right)_{N}\right)$ if and only if it belongs to $F\left(\left(\sim_{i}^{\prime}\right)_{N}\right)$. This completes our Step 1.

STEP 2: Fix $a, b \in M$. Consider two profiles of equivalence relations $\left(\sim_{i}\right)_{N}$ and $\left(\sim_{i}^{\prime \prime}\right)_{N}$ which satisfy the premises of binary independence axiom for $a$ and $b$. Now, consider the profile of equivalence relations $\left(\sim_{i}^{\prime}\right)_{N}$ as in Step 1. By Step 1, we get that $(a, b)$ belongs to $F\left(\left(\sim_{i}\right)_{N}\right)$ if and only if it belongs to $F\left(\left(\sim_{i}^{\prime}\right)_{N}\right)$ if and only if it belongs to $F\left(\left(\sim_{i}^{\prime \prime}\right)_{N}\right)$. Hence, $F$ satisfies binary independence.

Using Theorem 2 along with Lemma 1, the result now follows.

CASE 2: Suppose $m=2$. Let $M=\{a, b\}$. Call a set of attributes $S \subseteq N$ decisive if

- $a \sim b$ for all profiles $\left(\sim_{i}\right)_{N}$ such that $a \sim_{i} b$ for all $i \in S$

- and $a \nsim b$ for all profiles $\left(\sim_{i}\right)_{N}$ such that $a \nsim_{i} b$ for some $i \in S$,

where $\sim \equiv F\left(\left(\sim_{i}\right)_{N}\right)$. By Pareto $^{+}$, a decisive set exists. By Pareto ${ }^{-}$, the decisive set is non-empty. We will be done if we can show that the decisive set is unique. Suppose $S$ and $T$ are two different decisive sets. Consider a profile $\left(\sim_{i}\right)_{N}$ in which edge $(a, b)$ is present in $\sim_{i}$ if and only if $i \in S$. Similarly, consider a profile $\left(\sim_{i}^{\prime}\right)_{N}$ in which edge $(a, b)$ is present in $\sim_{i}^{\prime}$ if and only if $i \in T$. By definition, $\left(\sim_{i}\right)_{N} \neq\left(\sim_{i}^{\prime}\right)_{N}$ and $S \cap T \neq S$ or $S \cap T \neq T$. Since $S$ and $T$ are decisive sets, both $F\left(\left(\sim_{i}\right)_{N}\right)$ and $F\left(\left(\sim_{i}^{\prime}\right)_{N}\right)$ are complete equivalence relations (i.e., contain edge $(a, b))$. Hence, $F\left(\left(\sim_{i}\right)_{N}\right) \wedge F\left(\left(\sim_{i}^{\prime}\right)_{N}\right)$ is a complete equivalence relation. By definition, the profile $\left(\sim_{i}\right)_{N} \wedge\left(\sim_{i}^{\prime}\right)_{N}$ has edge $(a, b)$ only in attributes in $S \cap T$. By conjunctive separability $F\left(\left(\sim_{i}\right)_{N} \wedge\left(\sim_{i}^{\prime}\right)_{N}\right)=F\left(\left(\sim_{i}\right)_{N}\right) \wedge F\left(\left(\sim_{i}^{\prime}\right)_{N}\right)$. But $(S \cap T) \subsetneq S$. Since $S$ is decisive, this is a contradiction. 
Is Theorem 3 a tighter characterization of the family of meet aggregators than the one in Theorem 2? We try to answer this question. We give two examples to show that conjunctive separability and binary independence do not imply each other.

ExAmple 2 Fix $a, b \in M$. The aggregator $F$ is defined as follows. For all $c, d \in M$ where $(c, d) \neq(a, b)$ and for all profiles $\left(\sim_{i}\right)_{N}$, we have

- $c \nsim d$,

- and $a \sim b$ if and only if $a \sim_{1} b$ and $a \nsim_{i} b$ for all $i \neq 1$,

where $\sim \equiv F\left(\left(\sim_{i}\right)_{N}\right)$.

The aggregator $F$ in Example 2 satisfies binary independence. To see this, fix $x, y \in M$ and consider two profiles $\left(\sim_{i}\right)_{N}$ and $\left(\sim_{i}^{\prime}\right)_{N}$ as in the premises of the binary independence axiom. If $(x, y) \neq(a, b)$, then the edge $(x, y)$ is absent in both $F\left(\left(\sim_{i}\right)_{N}\right)$ and $F\left(\left(\sim_{i}^{\prime}\right)_{N}\right)$. If $(x, y)=(a, b)$, then clearly, the edge $(x, y)=(a, b)$ is present in $F\left(\left(\sim_{i}\right)_{N}\right)$ if and only if it is present in $F\left(\left(\sim_{i}^{\prime}\right)_{N}\right)$.

However, the aggregator $F$ in Example 2 fails conjunctive separability. An example is shown in Figure 3. We have only shown candidates $a$ and $b$ in Figure 3. The rest of the candidates can be put in any arbitrary equivalence class in each attribute. Figure 3 shows the output of the aggregator in three profiles, where the third profile is the meet of the first two profiles. It is easy to see that the aggregator violates conjunctive separability.

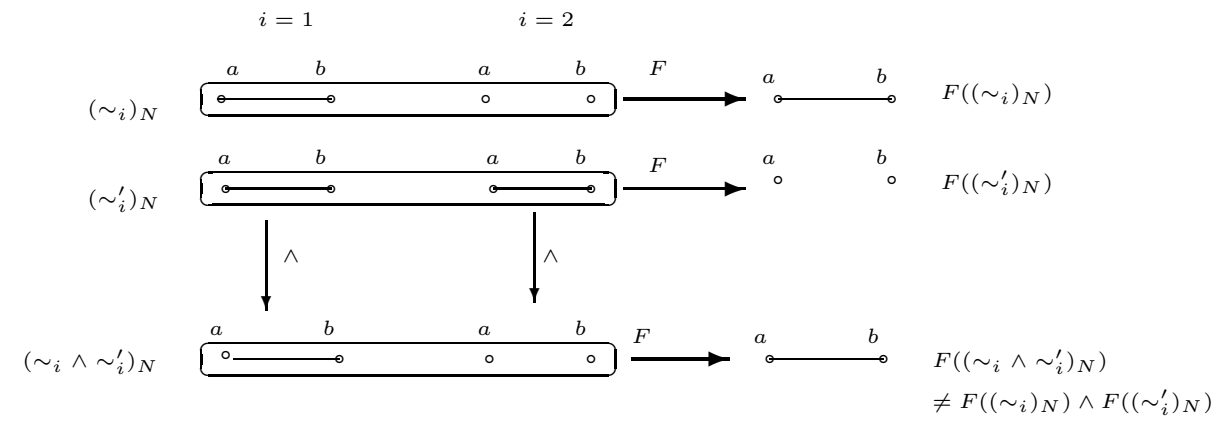

Figure 3: Binary independence does not imply conjunctive separability

The following example illustrates that conjunctive separability does not imply binary independence when $m \geq 3$.

ExAmple 3 Suppose $m \geq 3$. Define the aggregator $F$ as follows. For any profile $\left(\sim_{i}\right)_{N}$,

- if $\left(\sim_{i}\right)_{N}$ is a profile of complete equivalence relations, then $F\left(\left(\sim_{i}\right)_{N}\right)$ is a complete equivalence relation,

- else, it is the empty equivalence relation. 


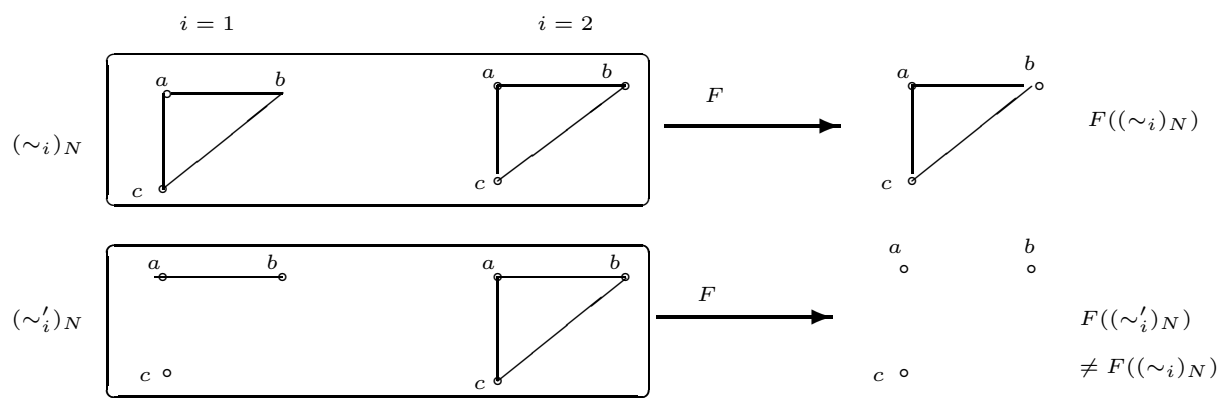

Figure 4: Conjunctive separability does not imply binary independence

Clearly, the aggregator in Example 3 satisfies conjunctive separability. Figure 4 shows two profiles where this aggregator fails binary independence.

Notice that the aggregators in Examples 2 and 3 do not satisfy Pareto ${ }^{+}$. This is no coincidence as the following proposition proves that conjunctive separability and Pareto ${ }^{+}$ are equivalent to binary independence and Pareto ${ }^{+}$if $m \geq 3$.

Proposition 1 Suppose $m \geq 3$ and let $F$ be an aggregator which satisfies Pareto ${ }^{+}$. The aggregator F satisfies conjunctive separability if and only if it satisfies binary independence.

Proof: We need the following lemma. The lemma is due to Fishburn and Rubinstein (1986), but we give a proof in the Appendix for completeness.

LEMMA 2 (Fishburn and Rubinstein (1986)) Suppose $m \geq 3$, and let $F$ be an aggregator which satisfies binary independence and Pareto ${ }^{+}$. Consider $a, b, c, d \in M$ and two profiles $\left(\sim_{i}\right)_{N}$ and $\left(\sim_{i}^{\prime}\right)_{N}$ such that for every $i \in N$, the edge $(a, b)$ belongs to $\sim_{i}$ if and only if the edge $(c, d)$ belongs to $\sim_{i}^{\prime}$. Then the edge $(a, b)$ belongs to $F\left(\left(\sim_{i}\right)_{N}\right)$ if and only if the edge $(c, d)$ belongs to $F\left(\left(\sim_{i}^{\prime}\right)_{N}\right)$.

Due to Lemma 1, we only have to prove that Pareto ${ }^{+}$and binary independence imply conjunctive separability. Assume for contradiction that conjunctive separability does not hold. Then, there are two profiles $\left(\sim_{i}\right)_{N}$ and $\left(\sim_{i}^{\prime}\right)_{N}$ and $a, b \in N$ such that either

a) the edge $(a, b)$ belongs to $F\left(\left(\sim_{i}\right)_{N}\right) \wedge F\left(\left(\sim_{i}^{\prime}\right)_{N}\right)$ but it does not belong to $F\left(\left(\sim_{i} \wedge \sim_{i}^{\prime}\right)_{N}\right)$,

b) the edge $(a, b)$ does not belong to $F\left(\left(\sim_{i}\right)_{N}\right) \wedge F\left(\left(\sim_{i}^{\prime}\right)_{N}\right)$ but it belongs to $F\left(\left(\sim_{i} \wedge \sim_{i}^{\prime}\right)_{N}\right)$.

Suppose (a) holds. Denote $F\left(\left(\sim_{i}\right)_{N}\right)$ as $\sim$ and $F\left(\left(\sim_{i}^{\prime}\right)_{N}\right)$ as $\sim^{\prime}$. Since the edge $(a, b)$ belongs to $\sim \wedge \sim^{\prime}$, it belongs to both $\sim$ and $\sim^{\prime}$. On the other hand the edge $(a, b)$ does not belong to $F\left(\left(\sim_{i} \wedge \sim_{i}^{\prime}\right)_{N}\right)$. By Pareto $^{+}$, there must exist some $i \in N$ such that the edge $(a, b)$ does not belong to $\sim_{i} \wedge \sim_{i}^{\prime}$. Define the following sets:

$$
\begin{aligned}
S & =\left\{i \in N \text { : edge }(a, b) \text { belongs to } \sim_{i}\right\} \\
S^{\prime} & =\left\{i \in N \text { : edge }(a, b) \text { belongs to } \sim_{i}^{\prime}\right\} \\
S^{\prime \prime} & =\left\{i \in N \text { : edge }(a, b) \text { belongs to } \sim_{i} \wedge \sim_{i}^{\prime}\right\} .
\end{aligned}
$$


By definition $S^{\prime \prime} \subseteq S$ and $S^{\prime \prime} \subseteq S^{\prime}$. We first show that $S$ and $S^{\prime}$ are distinct. Assume for contradiction $S=S^{\prime}$. Then, $S^{\prime \prime}=S=S^{\prime}$, and by binary independence, the edge $(a, b)$ must belong to $F\left(\left(\sim_{i} \wedge \sim_{i}^{\prime}\right)_{N}\right)$, which is a contradiction. Similarly, by binary independence again, $S$ and $S^{\prime}$ are strict supersets of $S^{\prime \prime}$, i.e., $S^{\prime \prime} \subsetneq S$ and $S^{\prime \prime} \subsetneq S^{\prime}$.

Consider a profile $\left(\dot{\sim}_{i}\right)_{N}$ and $c \in M \backslash\{a, b\}$ such that

$$
\begin{aligned}
& \left\{i \in N \text { : edge }(a, b) \text { belongs to } \dot{\sim}_{i}\right\}=S \\
& \left\{i \in N \text { : edge }(b, c) \text { belongs to } \dot{\sim}_{i}\right\}=S^{\prime} \\
& \left\{i \in N \text { : edge }(a, c) \text { belongs to } \dot{\sim}_{i}\right\}=S^{\prime \prime} .
\end{aligned}
$$

Note that $\left(\dot{\sim}_{i}\right)_{N}$ is well defined. Denote $F\left(\left(\dot{\sim}_{i}\right)_{N}\right)$ as $\dot{\sim}$. By binary independence, edge $(a, b)$ belongs to $\dot{\sim}$ since it belongs to $\sim$. By Lemma 2 , edge $(b, c)$ belongs to $\dot{\sim}$ since the edge $(a, b)$ belongs to $\sim^{\prime}$. By transitivity, edge $(a, c)$ belongs to $\dot{\sim}$. By Lemma 2 , the edge $(a, b)$ must then belong to $F\left(\left(\sim_{i} \wedge \sim_{i}^{\prime}\right)_{N}\right)$. This is a contradiction.

Assume now (b) holds. Since the edge $(a, b)$ does not belong to $\left(\sim \wedge \sim^{\prime}\right)$, it does not belong to $\sim$ or $\sim^{\prime}$. Without loss of generality, suppose $(a, b)$ does not belong to $\sim$. Define $S$ and $S^{\prime \prime}$ as in Equations 3 and 5 respectively. Since the edge $(a, b)$ belongs to $F\left(\left(\sim_{i} \wedge \sim_{i}^{\prime}\right)_{N}\right)$, by binary independence, $S^{\prime \prime} \subsetneq S$.

Consider a profile $\left(\dot{\sim}_{i}\right)_{N}$ and $c \in M \backslash\{a, b\}$ such that

$$
\begin{aligned}
\left\{i \in N \text { : edge }(a, b) \text { belongs to } \dot{\sim}_{i}\right\} & =S \\
\left\{i \in N \text { : edge }(b, c) \text { belongs to } \dot{\sim}_{i}\right\}=\left\{i \in N \text { : edge }(a, c) \text { belongs to } \dot{\sim}_{i}\right\} & =S^{\prime \prime} .
\end{aligned}
$$

Note that $\left(\dot{\sim}_{i}\right)_{N}$ is well defined. Denote $F\left(\left(\dot{\sim}_{i}\right)_{N}\right)$ as $\dot{\sim}$. By binary independence, the edge $(a, b)$ does not belong to $\dot{\sim}$ since it does not belong to $\sim$. But by Lemma 2 , the edges $(b, c)$ and $(a, c)$ belongs to $\dot{\sim}$ since $(a, b)$ belongs to $F\left(\left(\sim_{i} \wedge \sim_{i}^{\prime}\right)_{N}\right.$. By transitivity, $(a, b)$ belongs to $\dot{\sim}$. This is a contradiction.

Though conjunctive separability and binary independence are equivalent in the presence of Pareto ${ }^{+}$, conjunctive separability and binary independence are not equivalent in the presence of Pareto ${ }^{-}$. This is easily verified from Examples 2 and 3 (the aggregators in Examples 2 and 3 satisfy Pareto $^{-}$).

Proposition 1 is not true when $m=2$. In this case, we can easily construct rules which satisfies Pareto $^{+}$but does not satisfy conjunctive separability (and binary independence is satisfied vacuously). For example, the grand join aggregator satisfies Pareto ${ }^{+}$but fails conjunctive separability.

\subsection{Weakening Pareto ${ }^{-}$}

The characterizations in Theorems 2 and 3 use Pareto $^{-}$, a form of Pareto axiom which is not satisfied by the join aggregators. We wish to replace it with a weaker axiom which 
is satisfied by those aggregators. The following theorem shows that any aggregator which satisfies Pareto ${ }^{+}$and conjunctive separability must either be a meet aggregator or the trivial aggregator.

THEOREM 4 Consider the following statements.

1. An aggregator satisfies conjunctive separability, Pareto ${ }^{+}$, and non-triviality.

2. An aggregator satisfies binary independence, Pareto ${ }^{+}$, and non-triviality.

3. An aggregator belongs to the family of meet aggregators.

If $m \geq 3$, then (1), (2), and (3) are equivalent. If $m=2$ then (1) and (3) are equivalent.

Proof: Consider the case when $m \geq 3$. The fact that $(1) \Leftrightarrow(2)$ follows from Proposition 1 . Clearly, (3) implies (1) and (2). We show that (1) implies (3). We need the following lemma.

LEMma 3 Let $F$ be an aggregator which satisfies binary independence and Pareto ${ }^{+}$. Then, F satisfies neutrality.

Proof: For $m=2$, every aggregator satisfies neutrality, and hence the claim holds. For $m \geq 3$, the proof is almost immediate from Lemma 2. To see this, consider a permutation $\pi$ of the set of candidates and a profile $\left(\sim_{i}\right)_{N}$. Let $\left(\sim_{i}^{\pi}\right)_{N}$ be the permuted profile. Suppose $F$ does not satisfy neutrality. Then, some edge $(a, b)$ belongs to $F\left(\left(\sim_{i}\right)_{N}\right)$ but the edge $(\pi(a), \pi(b))$ does not belong to $F\left(\left(\sim_{i}^{\pi}\right)_{N}\right)$. Note that the profiles $\left(\sim_{i}\right)_{N}$ and $\left(\sim_{i}^{\pi}\right)_{N}$ satisfy the premises of Lemma 2 with edge $(\pi(a), \pi(b))$ taking the role of $(c, d)$. Hence, by Lemma 2 , the edge $(a, b)$ belongs to $F\left(\left(\sim_{i}\right)_{N}\right)$ if and only if the edge $(\pi(a), \pi(b))$ belongs to $F\left(\left(\sim_{i}^{\pi}\right)_{N}\right)$. This is a contradiction.

By Proposition 1 and Lemma 3, if an aggregator satisfies conjunctive separability and Pareto $^{+}$, then it satisfies neutrality. Now, consider an aggregator $F$ which satisfies conjunctive separability, Pareto ${ }^{+}$, and non-triviality. We now do the proof in two steps.

STEP 1: In this step, we show that if $\left(\sim_{i}\right)_{N}$ is a profile of empty equivalence relations then $F\left(\left(\sim_{i}\right)_{N}\right)$ is the empty equivalence relation. By neutrality, $F\left(\left(\sim_{i}\right)_{N}\right)$ is either the empty equivalence relation or the complete equivalence relation. Suppose it is the complete equivalence relation. Consider another profile $\left(\sim_{i}^{\prime}\right)_{N}$ such that $F\left(\left(\sim_{i}^{\prime}\right)_{N}\right)$ is not the complete equivalence relation. Such a profile exists because of non-triviality. But for every $i \in N$, $\sim_{i} \wedge \sim_{i}^{\prime}=\sim_{i}$. Hence, $F\left(\left(\sim_{i} \wedge \sim_{i}^{\prime}\right)_{N}\right)$ is a complete equivalence relation. By conjunctive separability, $F\left(\left(\sim_{i}^{\prime}\right)_{N}\right)$ is a complete equivalence relation. This is a contradiction.

STEP 2: In this step, we show that $F$ satisfies Pareto ${ }^{-}$, and by Theorem $3, F$ is an aggregator in the family of meet aggregators. To show that $F$ satisfies Pareto ${ }^{-}$, fix $a, b \in M$, and consider 
a profile $\left(\sim_{i}\right)_{N}$ such that the edge $(a, b)$ is not present in $\sim_{i}$ for all $i \in N$. Let $\left(\sim_{i}^{\prime}\right)_{N}$ be a profile of empty equivalence relations. These two profiles satisfy the premises in the binary independence axiom. Since by Step $1, F\left(\left(\sim_{i}^{\prime}\right)_{N}\right)$ is an empty equivalence relation, we get that the edge $(a, b)$ is not present in $F\left(\left(\sim_{i}\right)_{N}\right)$ because of binary independence ( $F$ satisfies binary independence by Proposition 1$)$. Hence, Pareto ${ }^{-}$holds.

Now, when $m=2$, we can go back to Case 2 in the proof of Theorem 3 . In the absence of Pareto ${ }^{-}$, we will either have a non-empty unique decisive set or an empty decisive set. An empty decisive set implies a trivial aggregator. Hence, by non-triviality, we must have a unique non-empty decisive set. The rest of the proof follows as in Case 2 in the proof of Theorem 3. Hence, (1) and (3) are equivalent for any $m \geq 2$.

The equivalence of (2) and (3) in Theorem 4 is a tighter characterization of the family of meet aggregators than the characterization of Fishburn and Rubinstein (1986) (Theorem 2). This is because non-triviality is weaker than Pareto ${ }^{-}$. For the same reason, the equivalence of (1) and (3) in Theorem 4 is a tighter characterization than Theorem 3.

\section{Duality in the Characterizations}

It is interesting to investigate if similar or "dual" characterizations are possible for the families of meet and join aggregators. The two main axioms, conjunctive and disjunctive separability, used in the characterizations in Theorems 1, 3, and 4 are dual to each other. However, we seem to require neutrality in Theorem 1, but not in Theorems 3 and 4 because it is implied by conjunctive separability and Pareto $^{+}$(Lemma 3$){ }^{3}$

One wonders if unanimity can be relaxed in the characterization of the join aggregators. For instance, does non-triviality, Pareto ${ }^{+}$, and disjunctive separability characterize the family of join aggregators? The answer is no. The aggregator in Example 1 satisfies all these axioms but it is not a join aggregator. Does neutrality, non-triviality, Pareto ${ }^{+}$, and disjunctive separability characterize this family? The answer is again no. The aggregator in the following example satisfies all these axioms but it is not a join aggregator.

EXAMPLE 4 The aggregator outputs the empty equivalence relation if the profile of equivalence relations include only empty equivalence relations, else it outputs the complete equivalence relation.

Another approach is to modify the characterizations of the family of meet aggregators to make it look analogous to Theorem 1. The following theorem attempts to do that.

THEOREM 5 An aggregator satisfies unanimity and conjunctive separability if and only if it belongs to the family of meet aggregators.

\footnotetext{
${ }^{3}$ This is similar to the Arrovian frameworks, where neutrality is implied by independence and Pareto axioms (Ubeda, 2004).
} 
Proof: Clearly, any meet aggregator satisfies unanimity and conjunctive separability. Now, let $F$ be an aggregator which satisfies unanimity and conjunctive separability. We show that $F$ satisfies Pareto $^{+}$.

Consider a profile $\left(\sim_{i}\right)_{N}$ such that the edge $(a, b)$ belongs to $\sim_{i}$ for all $i \in N$. Consider another profile $\left(\sim_{i}^{\prime}\right)_{N}$ such that for all $i \in N$, the only edge in equivalence relation $\sim_{i}^{\prime}$ is the edge $(a, b)$. By unanimity, the edge $(a, b)$ belongs to $F\left(\left(\sim_{i}^{\prime}\right)_{N}\right)$. Assume for contradiction that edge $(a, b)$ does not belong to $F\left(\left(\sim_{i}\right)_{N}\right)$. By conjunctive separability, the edge $(a, b)$ does not belong to $F\left(\left(\sim_{i} \wedge \sim_{i}^{\prime}\right)_{N}\right)$. Since for all $i \in N, \sim_{i} \wedge \sim_{i}^{\prime}=\sim_{i}^{\prime}$ and the edge belongs to $F\left(\left(\sim_{i}^{\prime}\right)_{N}\right)$, the edge must also belong to $F\left(\left(\sim_{i} \wedge \sim_{i}^{\prime}\right)_{N}\right)$. This is a contradiction.

Since $F$ satisfies unanimity, it satisfies non-triviality. Hence, $F$ satisfies conjunctive separability, Pareto ${ }^{+}$, and non-triviality, and by Theorem 4, it must belong to the family of meet aggregators.

Clearly, unanimity is weaker than the combination of Pareto ${ }^{+}$and Pareto $^{-}$. Hence, Theorem 5 is a tighter characterization than Theorem 3. Moreover, Theorem 5 gives us an almost dual characterization of the meet aggregators to the characterization in Theorem 1 of the join aggregators.

\section{Conclusion}

We conclude by giving two remarks on our characterizations.

AnONYMity. It is not difficult to see that adding anonymity to the list of axioms in Theorems 3, 4, and 5 give us the grand meet aggregator. It can be shown that anonymity is independent of the axioms used in these theorems. On the other hand, anonymity, unanimity, and disjunctive separability characterize the grand join aggregator. This can be seen from the proof of Theorem 3. We use neutrality in the proof of Theorem 3 to conclude $S^{a b}=S^{c d}$. But we can use anonymity instead of neutrality to conclude $S^{a b}=S^{c d}=N$. It is not difficult to argue that anonymity, unanimity, and disjunctive separability are independent axioms.

Dictatorial Aggregators. Call an aggregator $F$ dictatorial if there exists an attribute (dictator) $j \in N$ such that for every profile of equivalence relations $\left(\sim_{i}\right)_{N}$ we have $F\left(\left(\sim_{i}\right.\right.$ )$\left._{N}\right)=\sim_{j}$. Note that an aggregator $F$ is a dictatorial aggregator if and only if $F=F_{\vee}^{S}=F_{\wedge}^{S}$ for some $S \subseteq N$ and $|S|=1$. Thus, dictatorial aggregators are the only aggregators which belong to the families of meet and join aggregators. Using this fact and our results we can give various characterizations of the dictatorial aggregators. First, an aggregator is a dictatorial aggregator if and only if it satisfies conjunctive separability, disjunctive separability, Pareto ${ }^{+}$, and Pareto ${ }^{-}$. This follows from Theorems 1 and 3. Second, an aggregator is a dictatorial aggregator if and only if it satisfies conjunctive separability, disjunctive separability, Pareto ${ }^{+}$, and non-triviality. This follows from Theorems 1 and 4. Finally, an aggregator is a dictatorial 
aggregator if and only if it satisfies conjunctive separability, disjunctive separability, and unanimity. This follows from Theorems 1 and 5 .

\section{REFERENCES}

Ahn, D. S. And C. P. Chambers (2008): "What's on the Menu? Deciding What is Available to the Group?" Working Paper, California Institute of Technology.

Arrow, K. J. (1951): Social Choice and Individual Values, New York: Wiley, second Edition: 1963.

Barthélemy, J. P., , B. Leclerc, and B. Monjardet (1986): "On the Use of Ordered Sets in Problems of Comparison and Consensus of Classifications," Journal of Classification, 3, 187-224.

Barthélemy, J. P. (1988): "Comments on: Aggregation of Equivalence Relations by P.C. Fishburn and A. Rubinstein," Journal of Classification, 5, 85-87.

Çengelci, M. And R. Sanver (2010): "Simple Collective Identity Functions," Theory and Decision, 68, 417-443.

Chambers, C. P. And A. Miller (2010): "Group Signals," Working Paper, University of Haifa.

Dimitrov, D. And C. Puppe (2010): "Non-bossy Social Classification," Working Paper, Karlsruhe Institute of Technology.

Dimitrov, D., S.-C. Sung, And Y. Xu (2007): "Procedural Group Identification," Mathematical Social Sciences, 54, 137-146.

Fishburn, P. C. And A. Rubinstein (1986): "Aggregation of Equivalence Relations," Journal of Classification, 3, 61-65.

Geanakoplos, J. (2005): "Three Brief Proofs of Arrow's Impossibility Theorem," Economic Theory, 26, 211-215.

Hart, S. And A. Mas-Colell (1989): "Potential, Value and Consistency," Econometrica, $57,589-614$.

Houy, N. (2007): “'I want to be a J!": Liberalism in Group Identification Problems," Mathematical Social Sciences, 54, 59-70.

Kasher, A. And A. Rubinstein (1997): "On the Question "Who is a J?" A social Choice Approach," Logique et Analyse, 160, 385-395. 
LeClerC, B. (1984): "Efficient and Binary Consensus Functions on Transitively Valued Relations," Mathematical Social Sciences, 8, 45-61.

Miller, A. (2008): "Group Identification," Games and Economic Behavior, 63, 188-202.

Mirkin, B. (1975): "On the Problem of Reconciling Partitions," in Quantitative Sociology, International Perspectives on Mathematical and Statistical Modelling, ed. by H. M. Blalock, A. Aganbegian, F. Borodkin, R. Boudon, and V. Capecchi, Academic Press, New York, $441-449$.

Neumann, D. A. And V. T. Norton JR. (1986): "Clustering and Isolation in the Consensus Problem," Journal of Classification, 3, 281-297.

Perote-Pena, J. And A. Piggins (2002): "Geometry and Impossibility," Economic Theory, 20, 831-836.

Rubinstein, A. And P. C. Fishburn (1986): "Algebraic Aggregation Theory," Journal of Economic Theory, 38, 63-77.

Samet, D. And D. Schmeidler (2003): "Between Liberalism and Democracy," Journal of Economic Theory, 110, 213-233.

Shapley, L. (1953): Contributions to the Theory of Game II (Annals of Mathematics Studies 28), Princeton University Press, chap. A Value for n-Person Games, 307-317, ediors: H. W. Kuhn and A. W. Tucker.

UbedA, L. (2004): "Neutrality in Arrow and Other Impossibility Theorems," Economic Theory, 23, 195-204.

Wilson, R. (1978): "On the Theory of Aggregation," Journal of Economic Theory, 10, 89-99.

\section{APPENDIX}

\section{Aggregator in Example 1 Satisfies Disjunctive Separability}

Consider two profiles $\left(\sim_{i}\right)_{N}$ and $\left(\sim_{i}^{\prime}\right)_{N}$. We have to show that

$$
F\left(\left(\sim_{i} \vee \sim_{i}^{\prime}\right)_{N}\right)=F\left(\left(\sim_{i}\right)_{N}\right) \vee F\left(\left(\sim_{i}^{\prime}\right)_{N}\right)
$$

Suppose $(a, b)$ is an edge in $F\left(\left(\sim_{i} \vee \sim_{i}^{\prime}\right)_{N}\right)$. First, we show that $(a, b)$ in also an edge in $F\left(\left(\sim_{i}\right)_{N}\right) \vee F\left(\left(\sim_{i}^{\prime}\right)_{N}\right)$. We consider two cases.

CAse 1: Suppose $(a, b)$ is an edge in $\sim_{1} \vee \sim_{1}^{\prime}$. This in turn induces two subcases. 
- CASE 1A: The edge $(a, b)$ is in $\sim_{1}$ or in $\sim_{1}^{\prime}$. In such a case, the edge $(a, b)$ will either be in $F\left(\left(\sim_{i}\right)_{N}\right)$ or $F\left(\left(\sim_{i}^{\prime}\right)_{N}\right)$. Hence, it will be in $F\left(\left(\sim_{i}\right)_{N}\right) \vee F\left(\left(\sim_{i}^{\prime}\right)_{N}\right)$.

- CASE 1B: There is a chain of edges $\left(a, a_{1}\right),\left(a_{1}, a_{2}\right), \ldots,\left(a_{r}, b\right)$ which belongs to $\sim_{1}$ $\vee \sim_{1}^{\prime}$. Hence, these chain of edges are also present in $F\left(\left(\sim_{i}\right)_{N}\right) \vee F\left(\left(\sim_{i}^{\prime}\right)_{N}\right)$. Consequently, the edge $(a, b)$ is present in $F\left(\left(\sim_{i}\right)_{N}\right) \vee F\left(\left(\sim_{i}^{\prime}\right)_{N}\right)$.

CASE 2: Suppose $(a, b)$ is not an edge in $\sim_{1} \vee \sim_{1}^{\prime}$. Since $(a, b)$ is an edge in $F\left(\left(\sim_{i} \vee \sim_{i}^{\prime}\right)_{N}\right)$, there must exist $a_{0}, a_{1}, \ldots, a_{r}$ with $a_{0}=a$ and $b_{0}=b$ and $j_{1}, \ldots, j_{r} \in N$ such that for $k \in\{1, \ldots, r\}$, each edge $\left(a_{k-1}, a_{k}\right)$ belongs to $\left(\sim_{j_{k}} \vee \sim_{j_{k}}^{\prime}\right)$ or there is chain of edges $\left(a_{k-1}, c_{1}\right),\left(c_{1}, c_{2}\right), \ldots,\left(c_{q}, a_{k}\right)$ each of which belongs to $\left(\sim_{j_{k}} \vee \sim_{j_{k}}^{\prime}\right)$. By definition of the aggregator, each edge $\left(a_{k-1}, a_{k}\right)$ is in $F\left(\left(\sim_{i}\right)_{N}\right) \vee F\left(\left(\sim_{i}^{\prime}\right)_{N}\right)$. This in turn implies that $(a, b)$ is in $F\left(\left(\sim_{i}\right)_{N}\right) \vee F\left(\left(\sim_{i}^{\prime}\right)_{N}\right)$.

Suppose now $(c, d) \neq(a, b)$ is an edge in $F\left(\left(\sim_{i} \vee \sim_{i}^{\prime}\right)_{N}\right)$. Using a similar reasoning as above, we can conclude that $(c, d)$ is an edge in $F\left(\left(\sim_{i}\right)_{N}\right) \vee F\left(\left(\sim_{i}^{\prime}\right)_{N}\right)$.

Now, we show that if $(a, b)$ is an edge in $F\left(\left(\sim_{i}\right)_{N}\right) \vee F\left(\left(\sim_{i}^{\prime}\right)_{N}\right)$, then it is also an edge in $F\left(\left(\sim_{i} \vee \sim_{i}^{\prime}\right)_{N}\right)$. Again, there are multiple cases to consider.

CAse 1: The edge $(a, b)$ is in $F\left(\left(\sim_{i}\right)_{N}\right)$. So, either $(a, b)$ is an edge in $\sim_{1}$ or there is a chain of edges $\left(a, a_{1}\right),\left(a_{1}, a_{2}\right), \ldots,\left(a_{r}, b\right)$ which belongs to $\left(\sim_{i}\right)_{N}$. In the first case, the edge $(a, b)$ also belongs to $\left(\sim_{1} \vee \sim_{1}^{\prime}\right)$. Hence, it belongs to $F\left(\left(\sim_{i} \vee \sim_{i}^{\prime}\right)_{N}\right)$. In the second case, the same chain of edges belongs to $\left(\sim_{i} \vee \sim_{i}^{\prime}\right)_{N}$. Hence, $(a, b)$ belongs to $F\left(\left(\sim_{i} \vee \sim_{i}^{\prime}\right)_{N}\right)$.

CAse 2: The edge $(a, b)$ is in $F\left(\left(\sim_{i}^{\prime}\right)_{N}\right)$. Again, as in Case 1, we can argue that $(a, b)$ belongs to $F\left(\left(\sim_{i} \vee \sim_{i}^{\prime}\right)_{N}\right)$.

CASE 3: There is a chain of edges $\left(a, a_{1}\right),\left(a_{1}, a_{2}\right), \ldots,\left(a_{r}, b\right)$ which belongs to $F\left(\left(\sim_{i}\right)_{N}\right) \vee$ $F\left(\left(\sim_{i}^{\prime}\right)_{N}\right)$. Each of these edges can be supposed to be different from edge $(a, b)$. Consider any arbitrary edge $(c, d)$ in this chain. Edge $(c, d)$ is either in $\left(\sim_{i}\right)_{N}$ or in $\left(\sim_{i}^{\prime}\right)_{N}$ or there is a chain of edges $\left(c, c_{1}\right),\left(c_{1}, c_{2}\right), \ldots,\left(c_{q}, d\right)$ in $\left(\sim_{i} \vee \sim_{i}^{\prime}\right)_{N}$. In each of these cases, edge $(c, d)$ belongs to $\left(\sim_{i} \vee^{c} \sim_{i}^{\prime}\right)_{N}$. Since $(c, d) \neq(a, b)$, we conclude that $(c, d)$ belongs to $F\left(\left(\sim_{i} \vee^{c} \sim_{i}^{\prime}\right)_{N}\right)$. This is true for any edge in the chain $\left(a, a_{1}\right),\left(a_{1}, a_{2}\right), \ldots,\left(a_{r}, b\right)$. Hence, the edge $(a, b)$ belongs to $F\left(\left(\sim_{i} \vee^{c} \sim_{i}^{\prime}\right)_{N}\right)$

Finally, suppose $(c, d) \neq(a, b)$ is an edge in $F\left(\left(\sim_{i}\right)_{N}\right) \vee F\left(\left(\sim_{i}^{\prime}\right)_{N}\right)$. Using a similar reasoning as above, we can conclude that $(c, d)$ is an edge in $F\left(\left(\sim_{i} \vee \sim_{i}^{\prime}\right)_{N}\right)$.

Proof OF LemMa 2 
Proof: If $(a, b)=(c, d)$, then the claim is trivial. Assume $a, b, c$ are distinct. Consider two profiles $\left(\sim_{i}\right)_{N}$ and $\left(\sim_{i}^{\prime}\right)_{N}$ such that the edge $(a, b)$ is in $\sim_{i}$ if and only if the edge $(b, c)$ is in $\sim_{i}^{\prime}$. Consider another profile of equivalence relations $\left(\sim_{i}^{\prime \prime}\right)_{N}$ which satisfies the following for every $i \in N$ :

- the edge $(a, b)$ belongs to $\sim_{i}^{\prime \prime}$ if and only if it belongs to $\sim_{i}$,

- the edge $(b, c)$ belongs to $\sim_{i}^{\prime \prime}$ if and only if it belongs to $\sim_{i}^{\prime}$ (if and only if the edge $(a, b)$ belongs to $\sim_{i}$ ),

- and the edge $(a, c)$ belongs to $\sim_{i}^{\prime \prime}$.

Note that such a profile of equivalence relations $\left(\sim_{i}^{\prime \prime}\right)_{N}$ exists. By Pareto ${ }^{+}$, the edge $(a, c)$ belongs to $F\left(\left(\sim_{i}^{\prime \prime}\right)_{N}\right)$. By transitivity, the edge $(a, b)$ belongs to $F\left(\left(\sim_{i}^{\prime \prime}\right)_{N}\right)$ if and only if the edge $(b, c)$ belongs to $F\left(\left(\sim_{i}^{\prime \prime}\right)_{N}\right)$. By binary independence, edge $(a, b)$ belongs to $F\left(\left(\sim_{i}\right)_{N}\right)$ if and only if it belongs to $F\left(\left(\sim_{i}^{\prime \prime}\right)_{N}\right)$. Similarly, by binary independence again, edge $(b, c)$ belongs to $F\left(\left(\sim_{i}\right)_{N}\right)$ if and only if it belongs to $F\left(\left(\sim_{i}^{\prime \prime}\right)_{N}\right)$. Hence, edge $(a, b)$ belongs to $F\left(\left(\sim_{i}\right)_{N}\right)$ if and only if edge $(b, c)$ belongs to $F\left(\left(\sim_{i}^{\prime}\right)_{N}\right)$.

Now, consider a pair of profiles $\left(\sim_{i}\right)_{N}$ and $\left(\sim_{i}^{\prime}\right)_{N}$ such that for every $i \in N$, the edge $(a, b)$ belongs to $\sim_{i}$ if and only if the edge $(c, d)$ belongs to $\sim_{i}^{\prime}$. Now, construct $\left(\sim_{i}^{\prime \prime}\right)_{N}$ such that it satisfies the following: for every $i \in N$, the edge $(a, b)$ belongs to $\sim_{i}^{\prime \prime}$ if and only if the edge $(b, c)$ belongs to $\sim_{i}^{\prime \prime}$ if and only if the edge $(c, d)$ belongs to $\sim_{i}^{\prime \prime}$ if and only if the edge $(a, b)$ belongs to $\sim_{i}$ if and only if the edge $(c, d)$ belongs to $\sim_{i}^{\prime \prime}$. Applying the result in the previous paragraph multiple times, we get that the edge $(a, b)$ belongs to $F\left(\left(\sim_{i}\right)_{N}\right)$ if and only if the edge $(b, c)$ belongs to $F\left(\left(\sim_{i}^{\prime \prime}\right)_{N}\right)$ if and only if the edge $(c, d)$ belongs to $F\left(\left(\sim_{i}^{\prime}\right)_{N}\right)$. This proves the lemma. 